\title{
Retractile Proof Nets of the Purely Multiplicative and Additive Fragment of Linear Logic
}

\author{
Roberto Maieli ${ }^{\star}$ \\ Dipartimento di Filosofia \\ Università degli Studi "Roma Tre" \\ maieli@uniroma3.it
}

\begin{abstract}
Proof nets are a parallel syntax for sequential proofs of linear logic, firstly introduced by Girard in 1987 . Here we present and intrinsic (geometrical) characterization of proof nets, that is a correctness criterion (an algorithm) for checking those proof structures which correspond to proofs of the purely multiplicative and additive fragment of linear logic. This criterion is formulated in terms of simple graph rewriting rules and it extends an initial idea of a retraction correctness criterion for proof nets of the purely multiplicative fragment of linear logic presented by Danos in his Thesis in 1990.
\end{abstract}

\section{Introduction}

Proof nets are a parallel syntax (a graphical presentation) for sequential proofs of linear logic (LL), firstly introduced by Girard in [3]. An interesting challenge is to find intrinsic (geometrical) characterizations of proof nets, that is correctness criteria (naively, algorithms) for checking those proof structures which correspond to LL proofs; this is particularly true for proof nets of the pure multiplicative and additive fragment of linear logic (MALL).

Our starting idea is that correctness for MALL proof nets should be formulated as simple as possible, following the spirit of correctness for proof nets of the pure multiplicative fragment of linear logic (MLL, see [3] and [1). In our work correctness is formulated by an algorithm which implements simple graph rewriting rules. In particular, we extend an initial idea of a retraction correctness criterion for MLL proof nets presented in Danos's Thesis ([2]) and subsequently reformulated as a parsing criterion for MELL proof nets by Guerrini and Masini ([6]). Naively, retractility is a way to simulate sequentialization steps: each retracted (sub)graph corresponds to a correct (sequentializable) (sub)proof structure. Compared with other existing syntaxes for MALL proof nets, like that

* The author thanks Michele Abrusci, Paul Ruet and the anonymous referees for their suggestions and remarks. This work was partially supported by the MIUR Project Rete italo-francese di ricerca in logica e geometria della computazione (Prot. IIO4CE27L4). 
one due to Girard (4]) or Hughes-van Glabbeek ([7), our retractile correctness criterion does not rely on any notion of additive box, slice or jump. This effort should simplify the complexity of checking correctness. However here we do not discuss complexity aspects of our criterion; moreover, for simplicity reasons, we restrict to consider only cut-free proof nets.

After recalling, in next sub-section, some basic notions of the MALL fragment we introduce, in Section 2 a notion of (abstract) proof structure; then, in Section 3, we characterize correctness in terms of a rewriting algorithm which is shown confluent, correct (sequentializable) and complete (de-sequentializable) w.r.t. MALL sequent calculus. Finally, in Section 4, we discuss some directions in the way we could extend our criterion to proof nets with cuts.

\subsection{The MALL Fragment of Linear Logic}

MALL formulas $A, B, \ldots$ are built from literals (propositional variables $P, Q, \ldots$ and their negations $\left.P^{\perp}, Q^{\perp}, \ldots\right)$ by the binary connectives $\otimes$ (tensor), $>$ (par), $\&$ (with) and $\oplus$ (plus). Negation (. $)^{\perp}$ extends to arbitrary formulas by the de Morgan laws: $(A \otimes B)^{\perp}=\left(A^{\perp} 8 B^{\perp}\right),(A \otimes B)^{\perp}=\left(A^{\perp} \otimes B^{\perp}\right),(A \& B)^{\perp}=$ $\left(A^{\perp} \oplus B^{\perp}\right)$, and $(A \oplus B)^{\perp}=\left(A^{\perp} \& B^{\perp}\right)$. A MALL sequent $\Gamma$ is a non empty set of formula occurrences $A_{1}, \ldots, A_{n}$. We omit turnstiles $(\vdash)$ since all sequents are right-sided. Sequents are proved using the following rules:

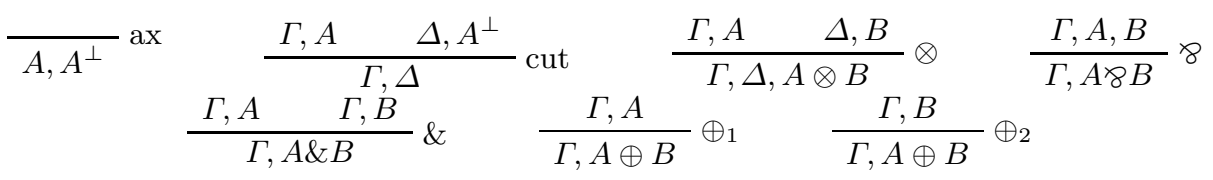

\section{Proof Structures}

Definition 1 (proof structure). A (cut-free) proof structure, shortly PS, of $M A L L$ is an oriented graph s.t. each edge is labelled by a MALL formula and built on the set of nodes (or vertices) following the typing constraints of Figure 1 . Pending edges are called conclusions. The orientation is from top to bottom; fixed a node, an entering edge is called premise while its unique emergent edge is called conclusion. We call link the graph made by a node together with its premise(s) and conclusion.

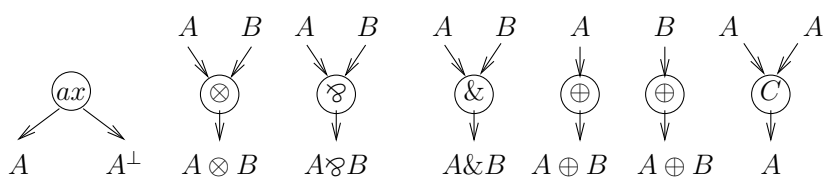

Fig. 1. MALL links 
Definition 2 (abstract proof structure). An abstract structure (AS) is a non oriented graph $G$ equipped with a set $\mathcal{C}(G)$ of pairwise disjoint pairs of coincident edges (two edges are coincident if they share at least a vertex). We call simply pair a pair of edges of $\mathcal{C}(G)$ and base of a pair (possibly one of) its common vertex(es). A pair is graphically denoted by a crossing arc close to the base.

An abstract proof structure (APS) is an AS such that:

- each edge is labelled by a MALL formula;

- each pair is denoted by an arc labelled by $8, \&$ or $C$;

- it is build by iterating the rules of Figure 2 (a mapping from PS to APS).
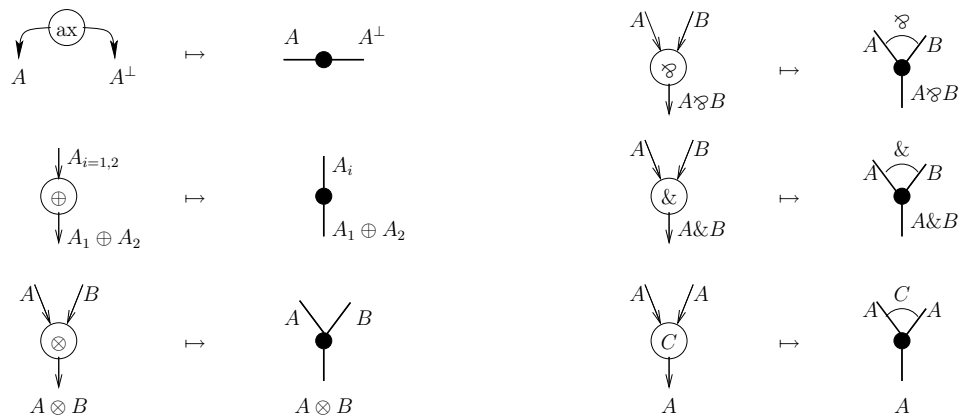

Fig. 2. Mapping PS in to APS

Notation: if $\pi$ is a PS then $\pi^{*}$ denotes its corresponding APS; variables $e_{1}, e_{2}, \ldots$ denote edges and $v_{1}, v_{2}, \ldots$ denote vertices of an APS; a dotted edge incident to a vertex $v$ and (eventually) labelled by variables $a, b, \ldots$, is a compact representation of possibly several edges incident to $v$; finally, $\delta(v)$ states for the degree (the number of incident edges) of a vertex $v$.

Definition 3 (multiplicative retraction). A multiplicative retraction of an APS $\pi$ is a rewriting of $\pi$ into $\pi^{\prime}$ (denoted $\pi \rightsquigarrow \pi^{\prime}$ ) by means of an instance of the following rules:

$R_{1}$ (on the left hand side of Figure [3), with the conditions that in $\pi$ :

- vertices $v_{1}$ and $v_{2}$ are distinct;

- the retracted edge $e_{1}$ does not belong to any pair of $\mathcal{C}(\pi)$.

$R_{2}$ (on the right hand side of Figure 3), with the conditions that in $\pi$ :

- vertices $v_{1}$ and $v_{2}$ are distinct;

- the two retracted edges $e_{1}$ and $e_{2}$ belong to the same 8 -pair.

Definition 4 (additive retraction)

An additive retraction of an APS $\pi$ is a rewriting of $\pi$ into $\pi^{\prime}$ by means of an instance of the following rules: 

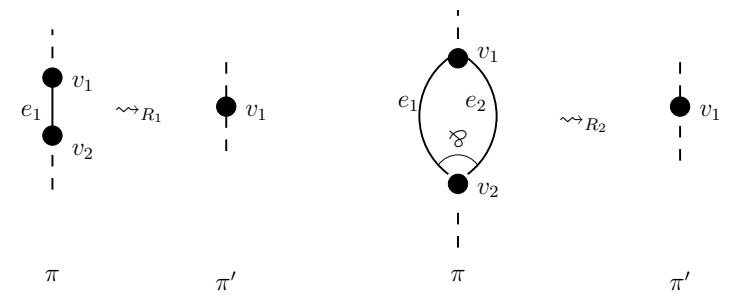

Fig. 3. Multiplicative retraction rules $R_{1}$ and $R_{2}$

$R_{3}$ (on the left hand side of Figure 4), with the conditions that in $\pi$ :

- each vertex $v_{i}, 1 \leq i \leq 4$, is distinct;

- the two retracted edges $e_{1}$ and $e_{2}$ belong to the same $C$-pair.

$R_{4}$ (on the right hand side of Figure 4), with the conditions that in $\pi$ :

- each vertex $v_{i}, 1 \leq i \leq 3$, is distinct;

- the two retracted edges $e_{1}$ and $e_{2}$ belong to the same \&-pair.

$-\delta\left(v_{2}\right)=1$ and $\delta\left(v_{3}\right)=1$.
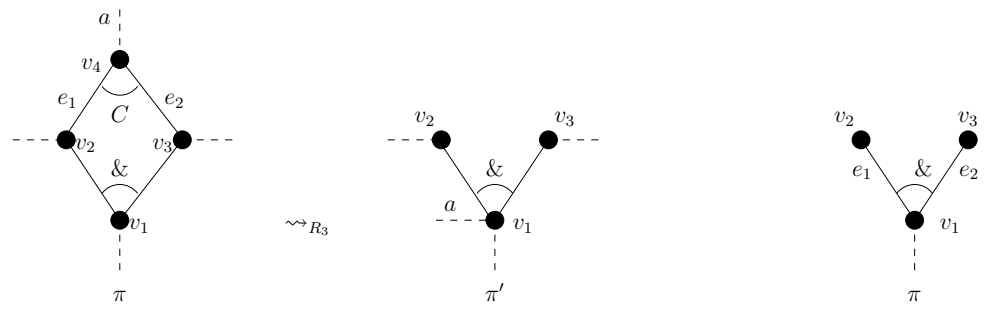

Fig. 4. Additive retraction rules $R_{3}$ and $R_{4}$

Definition 5 (distributive retraction). A distributive retraction rul 1 of an APS $\pi$ is a rewriting of $\pi$ into $\pi^{\prime}$ by means of an instance of the rule $R_{5}$ of Figure 5, with the conditions that in $\pi$ :

- each vertex $v_{i}, 1 \leq i \leq 8$, is distinct;

- the two retracted edges $e_{1}$ and $e_{2}$ belong to the same $C$-pair;

- the two retracted edges $e_{3}$ and $e_{4}$ belong to the same 8 -pair;

$-\delta\left(v_{4}\right)=2, \delta\left(v_{5}\right)=2, \delta\left(v_{6}\right)=3$ and $\delta\left(v_{7}\right)=3$.

Fixed a retraction rule $R_{i}, 1 \leq i \leq 5$, the subgraph of $\pi$ (resp., of $\pi^{\prime}$ ) depicted on the left (resp., on the right) hand side of the $\rightsquigarrow_{R_{i}}$ map is called the retraction (resp., retracted) graph of $R_{i}$.

We say that two instances $R_{i}$ and $R_{j}$, with $1 \leq i, j \leq 5$, overlap (resp., separate) when the intersection of their retraction graphs is not empty (resp.,

\footnotetext{
${ }^{1}$ This rule reflects the distributive linear law $(b \ngtr c) \&(b \ngtr d) \vdash b 8(c \& d)$.
} 

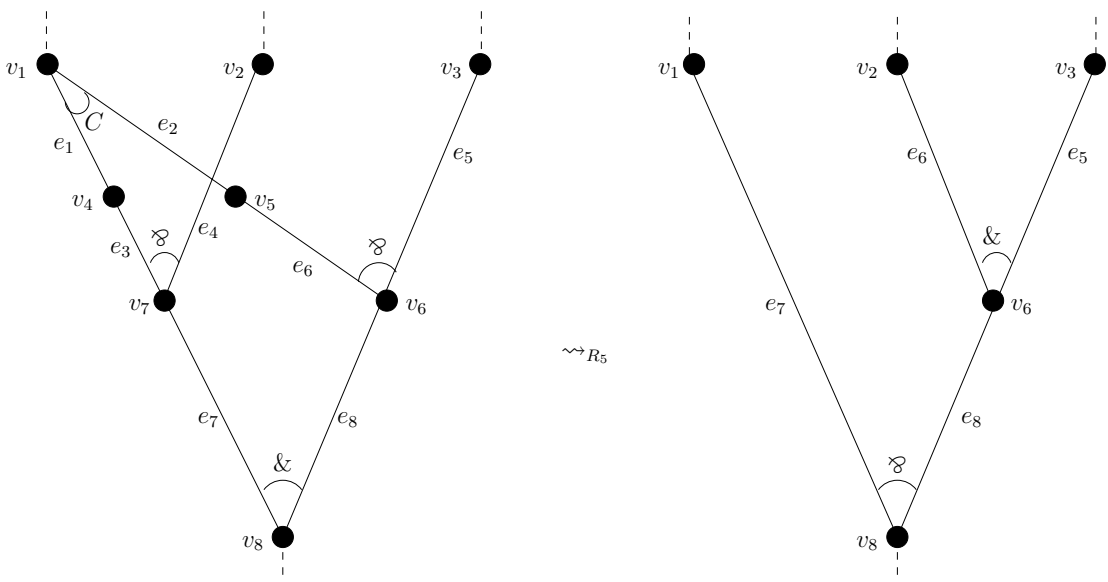

Fig. 5. Distributive retraction rule $R_{5}$

empty). $R_{i}$ and $R_{j}$ are said independent when they can be applied in any order, i.e. $R_{i}$ immediately before $R_{j}$ or $R_{j}$ immediately before $R_{i}$.

An APS $\pi$ with conclusions $A_{1}, \ldots, A_{n}$ is retractile when there exists a sequence of retraction instances starting with $\pi$ and terminating with a single node with $n$ incident edges labelled by $A_{1}, \ldots, A_{n}$.

Definition 6 (proof net). A PS $\pi$ with conclusions $A_{1}, \ldots, A_{n}$, with $n \geq 1$, is correct (i.e., it is a proof net) if its corresponding APS $\pi^{*}$ is retractile.

Theorem 1 (confluence). If $\pi$ is a retractile APS then any sequence of retraction instances starting from $\pi$ terminates with a single node with the (possibly) several incident edges labelled by the conclusions of $\pi$.

Proof. Assume $\Sigma$ is a retractions sequence $\pi \rightsquigarrow \pi_{1} \rightsquigarrow \ldots \rightsquigarrow \pi_{n}=\bullet$ with $R_{i}$ as first retraction (i.e. $\pi \rightsquigarrow R_{i} \pi_{1}$ ) and assume there exists a $\sigma$ such that $\pi \rightsquigarrow R_{j} \sigma$. We show that $\sigma$ is retractile too. We reason by induction on the length $l$ of $\Sigma$, where $l$ is the number of retraction instances of $\Sigma$.

Assume $l=1$, then $\pi \rightsquigarrow R_{i} \pi_{1}=\bullet$ and so $R_{i}$ and $R_{j}$ must be the same instance with $\sigma=\pi_{1}$. This follows from the definition of the retraction rules (if $R_{i}$ and $R_{j}$ are two different instances then the retraction graphs of $R_{i}$ and $R_{j}$ can be disjoint or partially overlapping but not included each other).

Assume $l>1$, then we split our reasoning in two sub-cases.

1. $R_{i}$ and $R_{j}$ are independent. Since, by assumption $\pi_{1}$ is retractile in $n-1$ steps, then by hypothesis of induction applied to $\pi_{1}$ we conclude that any $\pi_{1}^{\prime}$ obtained by $\pi_{1} \rightsquigarrow R_{j} \pi_{1}^{\prime}$ must be retractile. This means that $\sigma$ is retractile since $\sigma \rightsquigarrow R_{i} \pi_{1}^{\prime}$ and $R_{i}$ and $R_{j}$ are independent (see Figure 6).

2. $R_{i}$ and $R_{j}$ are not independent; this means that $R_{i}$ and $R_{j}$ must be two overlapping instances of the $R_{5}$ rule like in left hand side of Figure 7 . Again, 


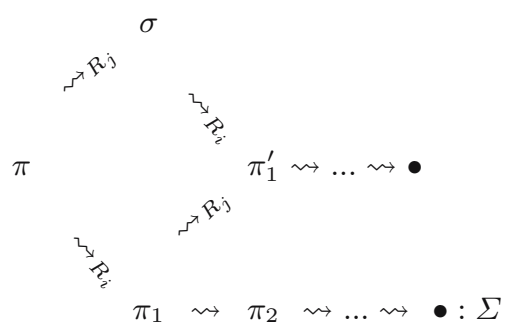

Fig. 6. Confluence of independent retractions

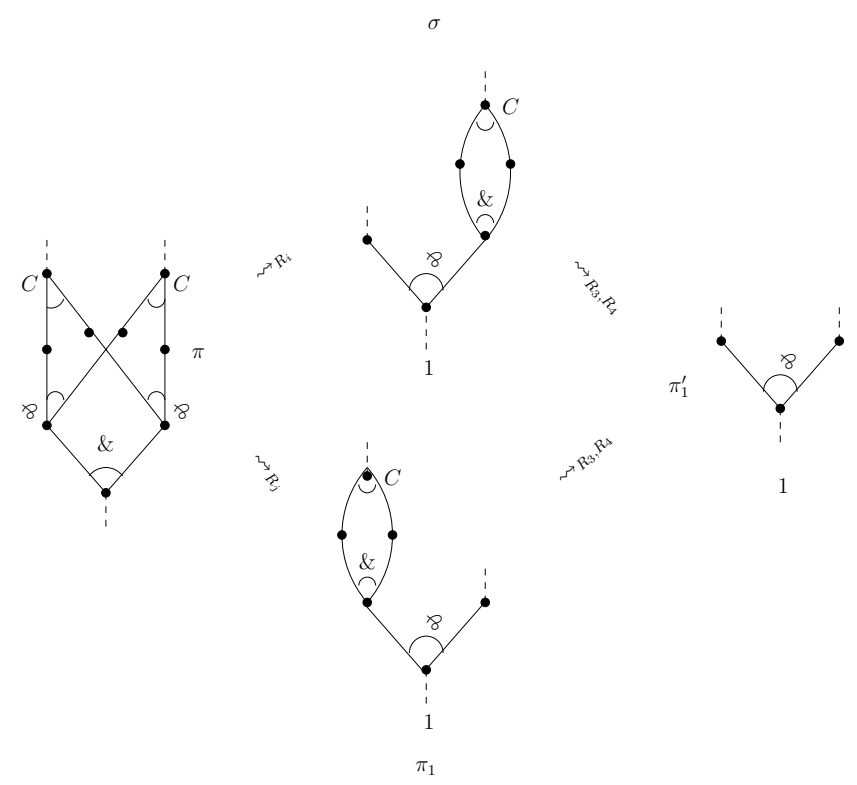

Fig. 7. Confluence of non independent retractions

we reason like in the previous case. Since, by assumption $\pi_{1}$ is retractile in $n-1$ steps, then we can apply the hypothesis of induction to $\pi_{1}$ and conclude that $\pi_{1}^{\prime}$ is also retractile since $\pi_{1} \rightsquigarrow R_{3}, 4 \pi_{1}^{\prime}$. This means that $\sigma$ will be retractile too, since $\sigma \rightsquigarrow R_{3}, 4 \pi_{1}^{\prime}$ (see Figure 6 ).

\section{3 (De-)Sequentialization}

In this section we show that any sequent proof of can be de-sequentialized into a proof net with the same conclusions (Theorem 2) and vice-versa (Theorem 5).

Theorem 2 (de-sequentialization). A proof $\pi^{-}$of the sequent $\Gamma=A_{1}, \ldots$, $A_{n \geq 1}$ can be desequentialized in to a proof net $\pi$ with conclusion $\Gamma$. 
Proof. By induction on the height of the given sequential proof $\pi^{-}$. We only consider the case when last rule of $\pi$ is a \&-rule (the other cases are very simple and we omit them). Assume $\pi^{-}$like in the left hand side of Figure 8, then by hypothesis of induction $\pi_{1}^{-}$and $\pi_{2}^{-}$desequentialize respectively into two retractile APS $\pi_{1}^{*}$ and $\pi_{2}^{*}$, like in the middle side of Figure 8, Clearly the resulting APS $\pi^{*}$ (see the right hand side of Figure 8) will be retracted to $\bullet$ by applying (iteratively) rule $R_{3}$ and (an instance of) rule $R_{4}$.
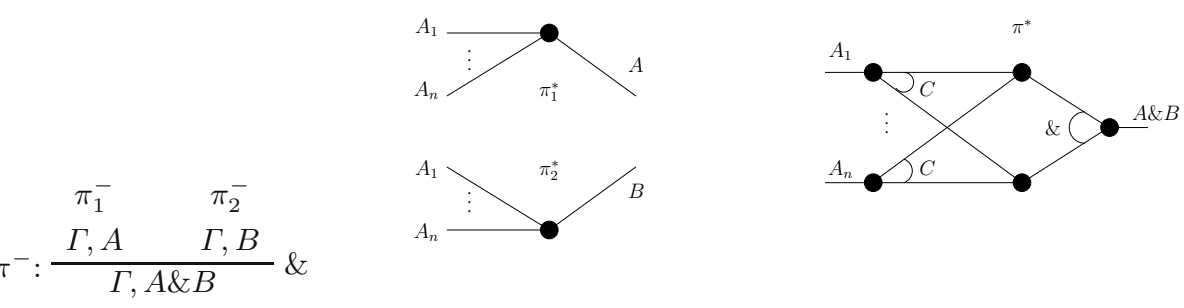

Fig. 8. De-sequentialization of the \&-rule

In the following we give an indirect proof of the sequentialization: first we show that any proof net can be weighted in such a way of becoming a proof net $\grave{a}$ la Girard (Section 3.1), then sequentialization follows as a consequence of Girard's one (Section 3.2).

\subsection{Girard's Proof Nets}

In this section we recall the basic notions of Girard's proof net; for simplification reasons we adopt the syntax of 9 .

Definition 7 (Girard's proof structure). A proof structure à la Girard (GPS) is a PS with weights associated as follows (weights assignment):

1. first we associate a boolean variable, called eigen weight $p$, to each \&-node (eigen weights are supposed to be different);

2. then we associate a weight, a product of (negation of) boolean variables $(p, \bar{p}, q, \bar{q} \ldots)$ to each node, with the constraint that two nodes have the same weight if they have a common edge, except when the edge is the premise of a $\&$ or $C$-node, in these cases we do like in Figure 9:

3. a conclusion node has weight 1 ;

4. if $w$ is the weight of a \&-node, with eigen weight $p$, and $w^{\prime}$ is a weight depending on $p$ and appearing in the proof structure then $w^{\prime} \leq w$ (we say that a weight $w$ depends on $p$ when $p$ or $\bar{p}$ occurs in $w)$.

${ }^{2}$ The height, $h\left(\pi^{-}\right)$, of $\pi^{-}$is defined inductively as usual. We consider last rule $R$ of $\pi^{-}$: if $R=a x$ then $h\left(\pi^{-}\right)=1$ otherwise if $R$ is an unary rule, 8 or $\oplus$, (resp., a binary rule, $\otimes$ or $\&$ ) then $h\left(\pi^{-}\right)=h\left(\pi_{1}^{-}\right)+1\left(\operatorname{resp} ., h\left(\pi^{-}\right)=\max \left(h\left(\pi_{1}^{-}\right), h\left(\pi_{2}^{-}\right)\right)+1\right)$ where $\pi_{1}^{-}$(resp., $\pi_{1}^{-}$and $\pi_{2}^{-}$) is the immediate sub-proof (resp., are the immediate sub-proofs) of $\pi^{-}$. 


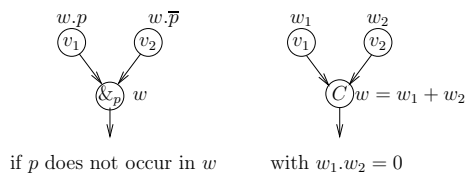

Fig. 9. Weights for GPS

A node $L$ with weight $w$ depends on the eigen weight $p$ if $w$ depends on $p$ or $L$ is a $C$-node and one of the weights just above it depends on $p$.

Definition 8 (slice and switchings). A valuation $\varphi$ for a GPS $\pi$ is a function from the set of all weights of $\pi$ into $\{0,1\}$. Fixed a valuation $\varphi$ for $\pi$ then:

- the slice $\varphi(\pi)$ is the graph obtained from $\pi$ by keeping only those nodes with weight 1 together its emerging edges;

- a multiplicative switching $S$ for $\pi$ is the non oriented graph built on the nodes and edges of $\varphi(\pi)$ with the modification that for each 8 -node we take only one premise (left/right 8 -switch);

- an additive switching (or simply a switching) is a multiplicative switching where for each \&-node we erase the (unique) premise in $\varphi(\pi)$ and we add an oriented edge, called jump, from the \&-node to an L-node whose weight depends on the eigen weight of the \&-node.

Definition 9 (Girard's proof net). A GPS $\pi$ is correct, so it is a proof net à la Girard (GPN), if any switching, induced by any valuation of $\pi$, is acyclic and connected (ACC).

Theorem 3 (sequentialization). A GPN can be sequentialized into a MALL sequent proof with same conclusions and vice-versa.

Proof. omitted (see[4]).

\subsection{Sequentialization}

Definition 10 (GAPS). A Girard abstract proof structure is an APS with weights associated as follows:

1. first we associate a distinct eigen weight variable to each \&-pair (graphically, the arc of each \&-pair is now labelled by a distinct eigen weight variable);

2. then we associate a weight to each node, with the constraint that two nodes have the same weight if they have a common edge, except when the edge occurs in a \&-pair or C-pair, in these cases we do like in Figure 10:

3. a conclusion node has weight 1 ;

4. if $w$ is the weight of a node that is the base of an $\&_{p}$-pair and $w^{\prime}$ is a weight depending on $p$ and appearing in the GAPS then $w^{\prime} \leq w$. 

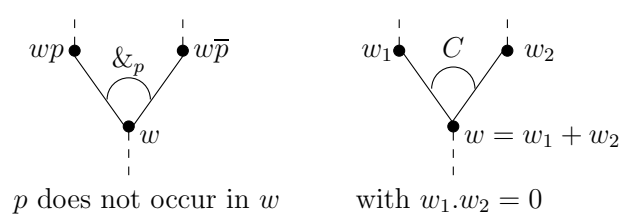

Fig. 10. Weights for GAPS

Remark 1. The notions of valuation and slice are still well defined w.r.t. GAPS. Only the definition of switching needs a slight modification. Fixed a valuation $\varphi$ for an GAPS $\pi$ then:

- a multiplicative switching $S$ for $\pi$ is the (non oriented) graph built on $\varphi(\pi)$ with the modification that for each 8 -pair we take only one edge (left/right 8-switch);

- an additive switching is a multiplicative switching where for each $\&_{p}$-pair we erase the (unique) edge in $\varphi(\pi)$ and we add a jump from the base of this $\&_{p}$-pair to an $L$-node whose weight depends on $p$.

Lemma 1. Assume $\pi$ is an APS such that $\pi \rightsquigarrow R_{i} \pi^{\prime}$, with $1 \leq i \leq 5$, and assume there exists a weights assignment making $\pi^{\prime}$ a GAPS, then this assignment can be extended in such a way to transform $\pi$ in to a GAPS.

Proof. We reason by cases, according to the retraction $R_{i}$.

Cases $R_{1}$. The weight $w$ associated to node $v_{1}$ in the retracted graph of $R_{1}$ in Figure 3 is inherited by both nodes $v_{1}$ and $v_{2}$ of the retraction graph. Since all other weights remain unchanged $\pi$ is a GAPS. Case $R_{2}$ is similar.

Case $R_{2}$ is similar.

Case $R_{3}$. Assume, in the retracted graph of Figure 4 $p$ is the eigen weight of the \&-pair, $w$ is the weight of node $v_{1}, w p$ is the weight of node $v_{2}$ and $w \bar{p}$ is the weight of node $v_{3}$. We can easily extend this weight assignment to the corresponding nodes of the retraction graph: it is easy to verify that this assignment preserves the property of being a AGPS w.r.t. $\pi$, since all pair of $\mathcal{C}(\pi)$ are pairwise disjoint and all other weights remain unchanged.

Case $R_{4}$. Assume node $v_{1}$ has weight $w$ in the retracted graph of Figure 4: then $w$ is trivially inherited by the corresponding node $v_{1}$ of the retraction graph. Now, chosen a new eigen weight $p$ for the \&-pair, we can assign weights $w p$ and $w \bar{p}$ to nodes, respectively, $v_{2}$ and $v_{3}$. Now, since all other weights remain unchanged, $\pi$ is a GAPS.

Case $R_{5}$. Assume a weight assignment for the retracted graph of Figure 5 as follows: the $\&_{p}$-pair together with nodes $v_{1}, v_{6}$ and $v_{8}$ have the same weight $w$, while nodes $v_{2}$ and $v_{3}$ have weights, respectively, $w p$ and $w \bar{p}$. This assignment can be easily extended to $\pi$ with a slight modifications: the \&-pair with base in $v_{8}$ inherits the eigen weight $p$ and $v_{8}$ inherits the weight $w$; then weight $w p$ is assigned to $v_{7}, v_{4}$ and $v_{2}$, and weight $w \bar{p}$ to $v_{6}, v_{5}$ and $v_{3}$; finally weight $w=w p+w \bar{p}$ is assigned to $v_{1}$. It is easy to verify that this new assignment 
preserves the property of being a AGPS w.r.t. $\pi$, since all pair of $\mathcal{C}(\pi)$ are pairwise disjoint and all other weights remain unchanged.

What follows is a well known graph theoretical property (see [5], pages 250-251) we will exploit in the proof of the Lemma 2

Property 1 (Euler-Poincaré invariance). Given a graph $\mathcal{G}$, then $\sharp C C-\sharp C y=$ $\sharp V-\sharp E$, where $\sharp C C, \sharp C y, \sharp V$ and $\sharp E$ denotes, respectively, the number of connected components, cycles, vertices and edges of $\mathcal{G}$.

We use the predicate $\operatorname{Gir}(\pi)$ for saying that a GAPS is correct in the sense of Definition 9, i.e., any switching $S(\pi)$, w.r.t. a fixed valuation $\varphi(\pi)$, is ACC.

Lemma 2. If $\pi$ is an GAPS and $\pi \rightsquigarrow R_{i} \pi^{\prime}, 1 \leq i \leq 5$, then $\operatorname{Gir}(\pi)$ if $\operatorname{Gir}\left(\pi^{\prime}\right)$.

Proof. Let us fix a valuation $\varphi$ for $\pi$. First observe that by Lemma 1 we have only to verify that every switching $S(\pi)$ is ACC. The proof idea, illustrated in Figure 11] relies on the fact that if $\pi \rightsquigarrow R_{i} \pi^{\prime}$ then any switching $S$ for $\pi$
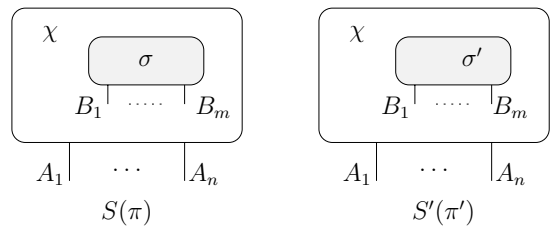

Fig. 11. Recovering $S(\pi)$ from $S^{\prime}\left(\pi^{\prime}\right)$

is nothing else that a switching $S^{\prime}$ for $\pi^{\prime}$ except for the fact we replace the switched retracted graph $\sigma^{\prime}$ of $R_{i}$ by a corresponding switched retraction graph $\sigma$. We reason by cases, according to the retraction rule $R_{i}$.

1. If $\pi r_{R_{1}} \pi^{\prime}$ (see the left hand side of Figure 3) then trivially any switching $S$ for $\pi$ can be recovered from a $S^{\prime}$ for $\pi^{\prime}$ where we replaced the retracted graph $\sigma^{\prime}$ with the retraction graph $\sigma$ of Figure 12. Clearly $S(\pi)$ is ACC.

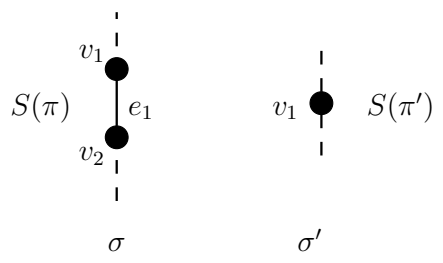

Fig. 12. Recovering $S(\pi)$ from $S^{\prime}\left(\pi^{\prime}\right)$ after a retraction $R_{1}$ 
2. If $\pi \rightsquigarrow R_{2} \pi^{\prime}$ (see the right hand side of Figure 3) then any switching $S$ for $\pi$ can be recovered from a switching $S^{\prime}$ for $\pi^{\prime}$ where we replaced the switched retracted graph $\sigma^{\prime}$ by a corresponding switched retraction graph $\sigma$ (resp., $\chi)$ in case we set a left (resp., right) switch for the 8-pair (see Figure 13). Clearly, $S_{\mho_{l}}(\pi)$ (resp., $\left.S_{\succ_{r}}(\pi)\right)$ is ACC.

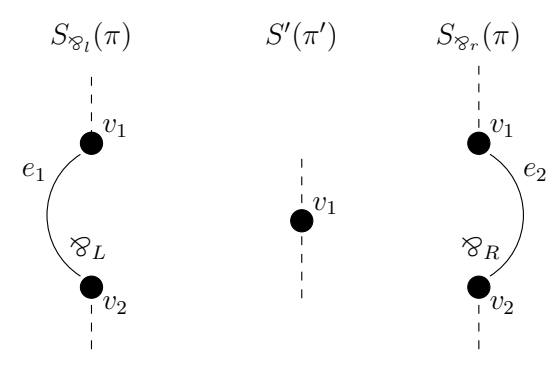

Fig. 13. Recovering $S(\pi)$ from ${ }^{\sigma^{\prime}} S^{\prime}\left(\pi^{\prime}\right)$ after ${ }^{\chi}$ a retraction $R_{2}$

3. If $\pi \rightsquigarrow R_{3} \pi^{\prime}$ (see the left hand side of Figure 4) then any switching $S$ for $\pi$ can be recovered from a switching $S^{\prime}$ of $\pi^{\prime}$. We need to consider two cases, according to the jump emerging from the base $v_{1}$ of the $\&_{p}$-pair in $S^{\prime}$.

First case: assume $S^{\prime}$ contains an immediate jump $j$ from the base $v_{1}$ of the $\&_{p}$-pair to its (unique) premise $v_{2}$ (see the right hand side of Figure 14). Then $S$ for $\pi$ can be recovered from $S^{\prime}$ where we replaced the switched retracted graph $\sigma^{\prime}$ by the corresponding switched retraction graph $\sigma$ on the left hand side of Figure 14. We have to show that $S$ is ACC. First, observe

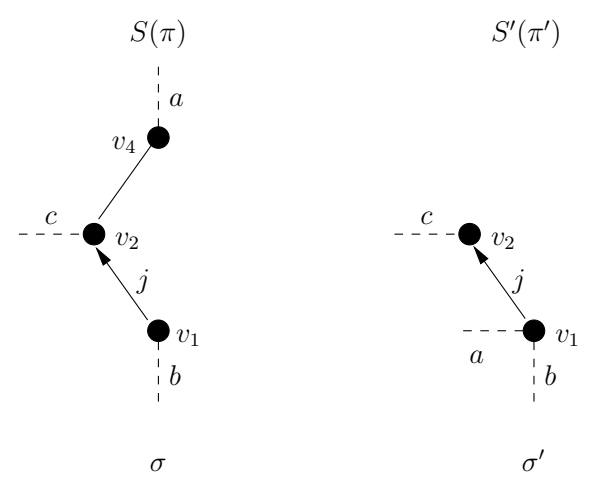

Fig. 14. Recovering $S(\pi)$ from $S^{\prime}\left(\pi^{\prime}\right)$, with an immediate jump, after a retraction $R_{3}$

that edges $a, b$ and $c$ are connected in $S$ as well in $S^{\prime}$, so the number of connected components in $S$ is 1 . Moreover in $S$ the difference $\sharp V-\sharp E$ must be the same as that one in $S^{\prime}$, that is 1 , since in $S$ there is only one more edge and one more vertex than in $S^{\prime}$. So by the Euler-Poincaré invariance in $S$ we have $\sharp C C-\sharp C y=1$, therefore $\sharp C y$ in $S$ must be 0 . So $S$ is ACC. 
Second case: $S$ contains a remote jump $j$ from the base $v_{1}$ of the $\&_{p}$ pair to a node $v$ depending on $p$ that is different from the (unique) premise of $\&_{p}$ (see the right hand side of Figure 15). Clearly $S$ can be recovered from
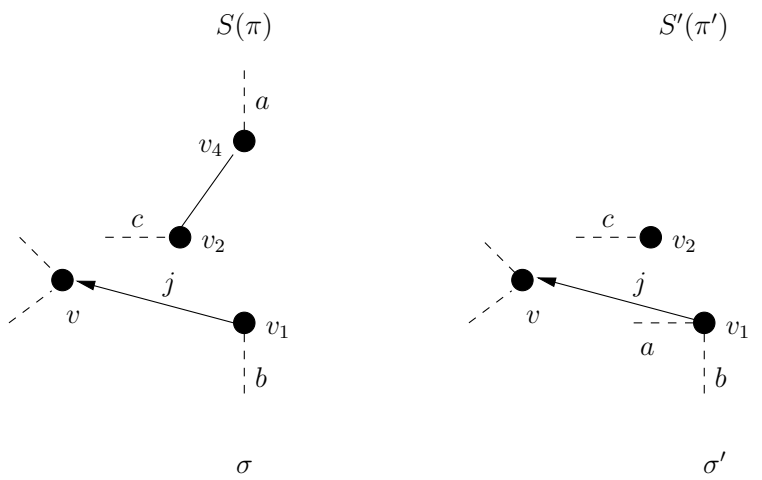

Fig. 15. Recovering $S(\pi)$ from $S^{\prime}\left(\pi^{\prime}\right)$, with a remote jump, after a retraction $R_{3}$

a switching $S^{\prime}$ where we replaced the switched retracted graph $\sigma^{\prime}$ by the switched retraction graph $\sigma$ on the left hand side of Figure 15. We show that $S$ is ACC. Although vertices $v_{1}$ and $v_{2}$ are connected in $S^{\prime}$ by assumption, they cannot be connected through $a$, otherwise we could easily set a switching $S^{\prime \prime}$ for $\pi^{\prime}$ that is identical to $S^{\prime}$ except for the immediate jump from $v_{1}$ to $v_{2}$ and get a cycle, contradicting the assumption $\operatorname{Gir}\left(\pi^{\prime}\right)$. This means that the retraction step $R_{3}$ preserves backwards the connection of $S$, so $\sharp C C$ of $S$ is 1. Now, observe that the difference $\sharp V-\sharp E$ of $S$ is the same as that one of $S^{\prime}$ (i.e. 1), so by the Euler-Poincaré invariance we have, in $S, \sharp C C-\sharp C y=1$. This means that, in $S, \sharp C y$ must be 0 . So $S$ is ACC.

4. If $\pi \rightsquigarrow R_{4} \pi^{\prime}$ (see the right hand side of Figure 4) then a switching $S$ for $\pi$ can be recovered from a switching $S^{\prime}$ for $\pi^{\prime}$ plus a jump $j$ emerging from the base $v_{1}$ of the $\&_{p}$ pair. Now, observe this jump $j$ in $S$ can only be directed to its (unique) premise, otherwise: $(i)$ either there would exist in $\pi^{\prime}$ a node whose weight depends on a variable $p$ that is not an eigen weight of any \&-pair or ( $i$ i) there would exist in $\pi$ two \&-pairs with the same eigen-weight variable $p$. Both cases contradict that $\pi$ is an AGPS (Lemma 1).

5. If $\pi \rightsquigarrow R_{5} \pi^{\prime}$ (see Figure 5) then a switching $S$ for $\pi$ is exactly a switching $S^{\prime}$ for $\pi^{\prime}$ except for the the jump emerging from the $\&_{p}$-pair and the switch for the 8 pair occurring in the retraction $R_{5}$. So, let us fix in $S^{\prime}$ a left 8 -switch and jump from the base $v_{6}$ of the $\&_{p}$-pair (the case with the right 8 -switch, is analogous): there are two possible jumps.

First case: assume in $S^{\prime}\left(\pi^{\prime}\right)$ we jump from the base $v_{6}$ of the $\&_{p}$ pair to its (unique) premise. Then a switching $S$ for $\pi$ is exactly a switching $S^{\prime}$ for $\pi^{\prime}$ except for the fact we replaced the switched retracted graph $\sigma^{\prime}$ with $\sigma$ of Figure 16. Clearly $S$ is connected, so via the Euler-Poincaré invariance, we conclude that $S$ is also acyclic (we reason like in the previous point 3 ). 

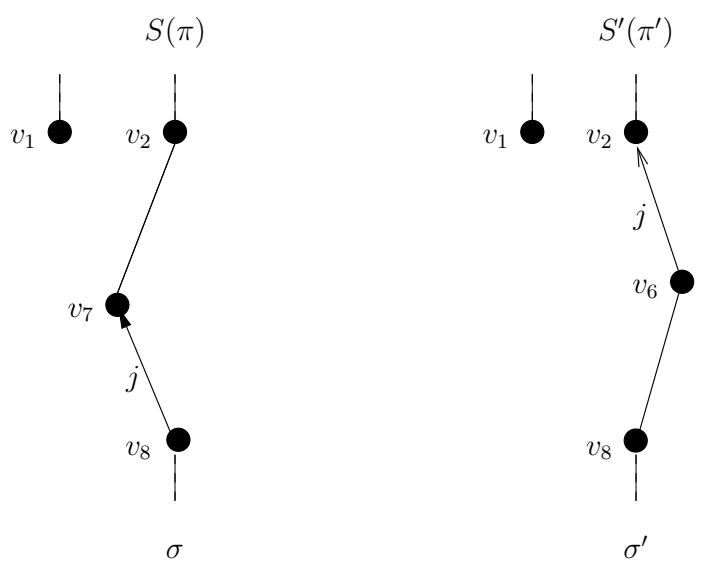

Fig. 16. Recovering $S(\pi)$ form $S^{\prime}\left(\pi^{\prime}\right)$, with immediate jump, after a reduction $R_{5}$

Second case: assume in $S^{\prime}(\pi)$ we jump from the base $v_{6}$ of the $\&_{p}$ pair to a remote node $v$ depending on $p$. Then a switching $S$ for $\pi$ can be recovered from a switching $S^{\prime}$ where we replaced the switched retracted graph $\sigma^{\prime}$ with $\sigma$ of Figure 17. Now observe that $\sigma^{\prime}$ does not induce any cycle in $S$, otherwise
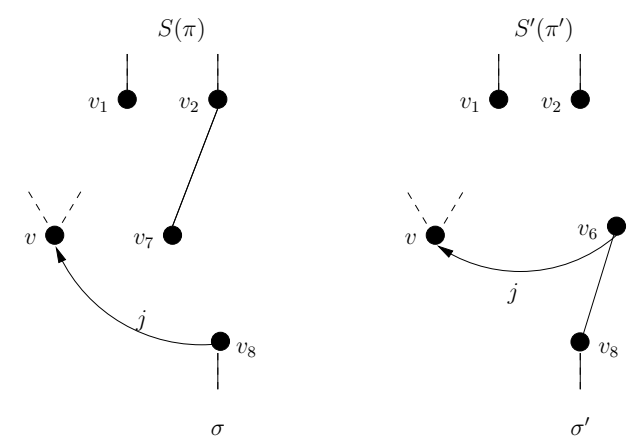

Fig. 17. Recovering $S(\pi)$ form $S^{\prime}\left(\pi^{\prime}\right)$, with a remote jump, after a reduction $R_{5}$

this cycle would already occur in $S^{\prime}$. Moreover, the number of vertices and edges in $S$ is the same as in $S^{\prime}$ so, via the Euler-Poincaré invariance, we conclude that $S$ is connected.

Theorem 4 ( $\mathbf{P N} \mapsto \mathbf{G P N})$. If $\pi$ is a PN then there exists a weight assignment transforming $\pi$ in to a GPN.

Proof. If $\pi$ is a PN then $\pi^{*}$ retracts to a node $v$ with possibly several incident edges labelled by the conclusions of $\pi$. Trivially $v$ is an AGPS satisfying the predicate Gir, then by iteration of Lemma 2 we conclude that $\pi$ is a GPN. 
Theorem 5 (sequentialization). If $\pi$ is a PN with conclusions $\Gamma$ then the sequent $\Gamma$ is provable in the MALL sequent calculus.

Proof. It follows from Girard's sequentialization (see 4) via Theorem 4]

\section{Conclusions and Forthcoming Work}

We presented a simple system of graph rewriting rules which can be viewed as a geometrical correction criterion for cut free proof structures of MALL. Each proof structure that is correct in our sense it will be so also in Girard's sense but not vice-versa. In general the other direction of Theorem 4 does not hold: the proof structure $\pi_{1}$, on the left hand side of Figure 18 is not correct for us (it is not retractile); nevertheless we can find a weight assignment transforming $\pi_{1}$ in to a Girard proof net. Actually we only accept correct the proof structure $\pi_{2}$ depicted on the right hand side of Figure 18 which (in our opinion) better embeds the two different sequentializations induced by the permutability of the $\&$-rule w.r.t. the $\otimes$-rule of the sequent calculus.
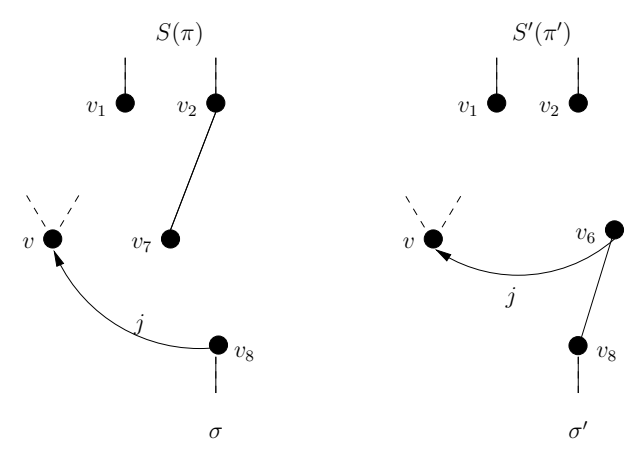

Fig. 18. Examples of PS w.r.t. sequentialization

As future work we aim at comparing the complexity of the retractility correctness criterion w.r.t. Girard and Hughes-van Glabbeek's criteria. Moreover we aim at extending retraction rules in such a way to take in to account proof nets with cuts. At the moment we are investigating some local (commutative) cut reduction steps, following the style of the Interaction Nets (8). Our idea is sketched in Figure 19 where the $\star$ symbol states for a binary MALL connective

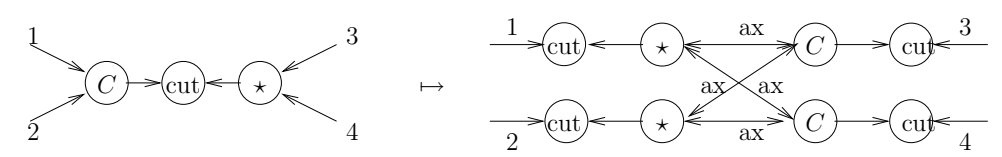

Fig. 19. Commutative cut step reduction 
or a contraction $C$. But in that case the correspondence with monomial GPS is lost: as soon as we replace the $\star$ symbol with the \& connective we are suddenly faced to proof structures weighted with polynomials.

\section{References}

1. Danos, V., Regnier, L.: The Structure of Multiplicatives. Archive for Mathematical Logic 28, 181-203 (1989)

2. Danos, V.: La Logique Linéaire appliquée à l'étude de divers processus de normalisation (principalment du $\lambda$-calcul). PhD Thesis, Univ. Paris VII (Juin 1990)

3. Girard, J.-Y.: Linear Logic. Theoretical Computer Science 50, 1-102 (1987)

4. Girard, J.-Y.: Proof nets: the parallel syntax for proof theory. In: Logic and Algebra, Marcel Dekker (1996)

5. Girard, J.-Y.: Le point aveugle. In: Hermann (ed.) Cours de Logique. Vers la Perfection, Paris, vol. I (2006)

6. Guerrini, S., Masini, A.: Parsing MELL proof nets. Theoretical Computer Science 254, 317-335 (2001)

7. Hughes, D., van Glabbeek, R.: Proof Nets for Unit-free Multiplicative-Additive Linear Logic. In: Proc. of IEEE Logic in Computer Science, IEEE Computer Society Press, Los Alamitos (2003)

8. Lafont, Y.: From proof nets to interaction nets. In: Girard, J.-Y., Lafont, Y., Regnier, L. (eds.) Advanced in Linear Logic, pp. 225-247. Cambridge Press, Cambridge (1995)

9. Laurent, O.: Polarized Proof Nets: Proof Nets for LC (Extended Abstract). In: Girard, J.-Y. (ed.) TLCA 1999. LNCS, vol. 1581, pp. 213-227. Springer, Heidelberg (1999) 